\title{
Substructure Shape Analysis for Kanji Character Recognition
}

\author{
Jairo Rocha and Hiromichi Fujisawa* \\ Central Research Laboratory, Hitachi, Ltd. \\ Kokubunji-shi, Tokyo, 185, Japan
}

\begin{abstract}
A method towards analytical recognition of Chinese characters is described. Basic character components (substructures) of any size are recognized anywhere on a Kanji string, even if they touch other components. The algorithm performs skeleton extraction, skeleton grouping, indexing of structural features on a previously generated look-up table, structural verification of hypothesis using model graphs, and geometrical verification by an array of neural nets, each one specialized on the geometry of each model. The system retrieves $98 \%$ of substructures with $91 \%$ precision rate.
\end{abstract}

\section{Introduction}

According to $[9,11]$, there are several basic formation rules for Chinese characters. A Chinese character is formed by a number of components, which are made of a number of strokes. See Figure 1. Thus, a Chinese character can be described in a three-level representation hierarchy, this is the character level, the component level, and the stroke level.

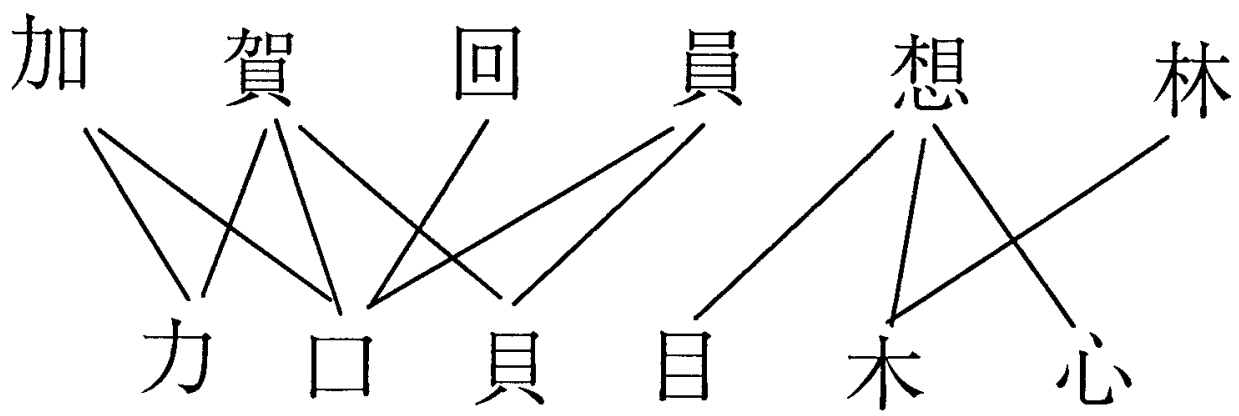

Fig. 1. Characters and components.

There are approximately 400 basic components and some 20 essential strokes. These numbers are much less than the total number of Chinese characters, which is over 50,000 . Hence, nobody denies that a hierarchical representation would

* e-mails: jairo@ipc4.uib.es, fujisawa@crl.hitachi.co.jp 
simplify the task of character definition. However, the use of a hierarchical representation for recognition is seldom reported because the components of a Kanji character may be difficult to separate from the others during an attempt to recognize individual components of a character. In [11], for example, it is assumed that the top level representation of a character into its components can be obtained using the white space between them.

Babaguchi et al. [1] and Cheng et al. [4] tried to extract substructures from characters but assuming fix positions for them, or using temporal information for on-line recognition.

We do know of any other report in the literature that attempts substructure recognition. The reason, as it was said before, is maybe that it is as difficult to recognize a substructure as to recognize the whole character, because of substructure inter-connections, and substructures made of disconnected parts. Strategies for recognition of individual characters report above $95 \%$ on printed characters [5], and from $85 \%$ to $95 \%$ on hand-printed characters [7].

We present in this paper a proof of concept on the possibility to recognize substructures anywhere in a group of characters, of any size, or connected to other character parts. To do so, we have employed scene analysis techniques [10], in other words, we spot substructures as 2-D objects on an image, allowing the objects to interact by touching each other. These complex techniques allow the use of general computer vision strategies. However, we are far from having a complete system. The reason for this is that we believe that the successful recognition of Kanji characters by subpart analysis implies the solution of most representational and computational problems on 2-D computer vision. This is another good reason to study the challenging problem of recognition and interaction of substructures on Chinese characters.

In the following section, we describe the different modules of the system, which includes the following novelties: an analytical approach to Chinese character recognition, an extension of the homeomorphic matching concept [13] and its implementation, and the use of an array of neural nets to accomplish detailed geometrical decisions, subordinated to the structural matching of model graphs.

\section{Overview}

A system is under development to implement the component recognition strategy. The basic modules of the system, as shown in Figure 2 are skeleton extraction, grouping of segments into paths, indexing of triplets of paths, consistency clustering of votes, and geometric consistency checking. Skeleton segments that are approximately collinear are grouped into paths that may be interpreted as character strokes. Triplets of connected paths are used as descriptive features that suggest model hypothesis. Different hypothesis are combined to create consistent matchings between input subgraphs and models. Structural matchings are further tested for geometrical quality regarding length proportions, angles and position of relative connections. 


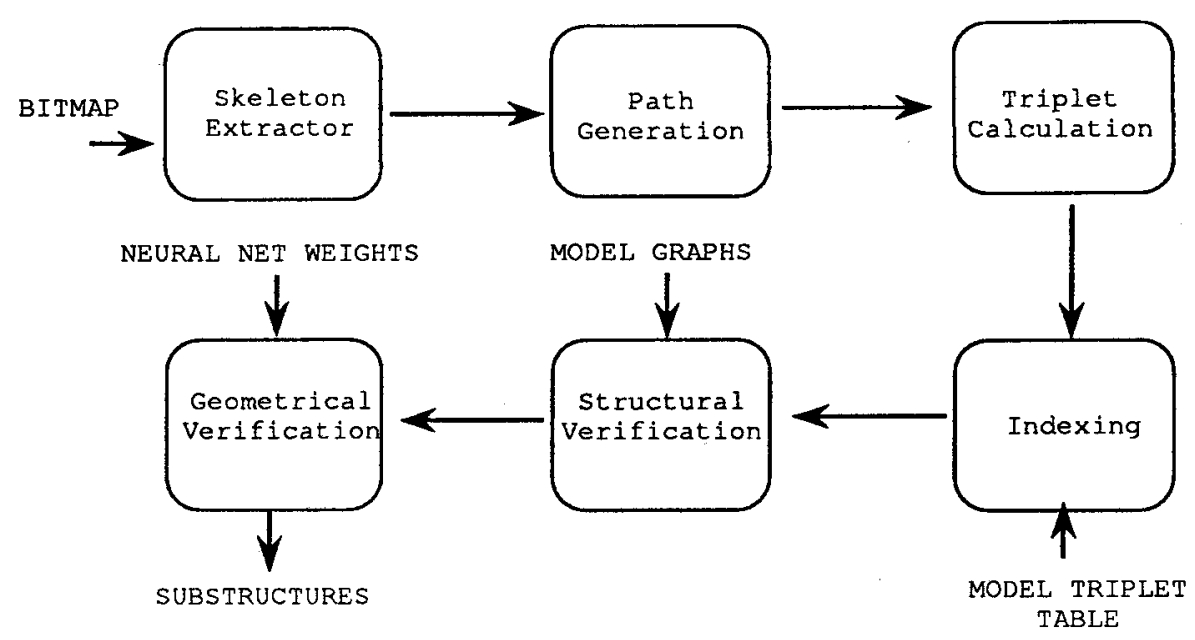

Fig. 2. System modules.

The system is used in two modes: training and testing. During the training mode, the images of the Kanji components displayed in Figure 3 are scanned, skeletons are extracted and paths are generated. Also, all triplets of paths are generated for model graphs as it is described later in the paper. Triplets and their distortions are stored in a triplet look-up table that will be used during the testing mode. The names of the structures in Figure 3 are from left to right: kuchi, ki, yama, kanmuri, shi, sa, oh, un, mata, and kozato .

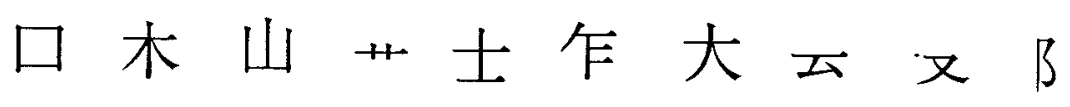

Fig. 3. Images for the models used by the system.

Once model graphs are included and the look-up table is filled, the system can retrieve substructures of real characters. The system is used again on training mode when a human trainer associates quality values to substructures retrieved from training characters. These values are used to train statistical classifiers designed to decide on the geometrical quality of characters during testing.

In short, we describe in the following sections an implementation that can recognize 10 substructures on printed characters. The method is bottom-up so it can be extended to recognize 400 substructures without having to search for each model instance in the input. However, several assumptions are made in order to reduce the implementation time so that the major techniques are tested. The simplifications are: connectivity relations are the only ones used, although the system could handle others like nearness; approximate collinearity 
and good continuity are supposed to generate the stroke paths needed, so major distorsions of paths are not handled bottom-up.

\section{Path Generation}

Segments may need to be associated before matching, and our system uses some criteria including relative collinearity of adjacent segments, and good continuity in joints [2]. In a joint, the two most collinear segments are associated, if the angle between them is less than 40 degrees. This association is repeated until there are no more segments or they are no collinear enough. Then, all associated segments are gather into paths. Figure 4 shows an example.
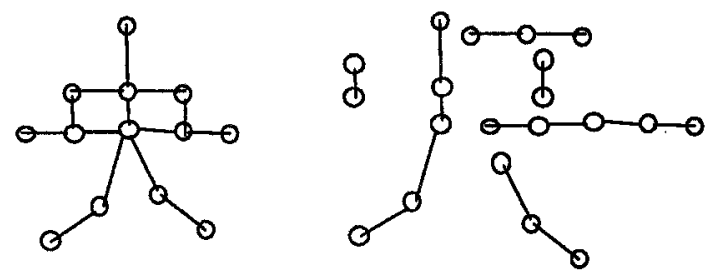

Fig. 4. Maximal paths of a skeleton graph.

Even if two segments are perfectly collinear, they may not need to be associated because the correct interpretation of the character requires them to be separated. Therefore, all possible subpaths of each path are considered.

The path generation process finds approximate collinear paths that are as long as possible and are called maximal paths. Then, for each maximal path, it generates all possible subpaths, which will be called simply paths of the graph, since no other graph paths will be considered in this paper. Note that any segment is a path; conversely, any path is made of approximately collinear segments between its ending joints, and, it makes sense to associate a length and an angle to it. It was found experimentally that the average number of segments in a skeleton (on 100 characters) is 21.6. Also, the average number of paths was 33.5, which shows that considering all subpaths is not a real computational burden.

Two paths that do not share segments are called compatible.

Two compatible paths may be spatially related according to their connections: one path may intersect another one on its Head, Tail or Inside, and this relation is calculated for all compatible paths as an extension of the adjacency relation of the raw segments.

In summary, an extended graph $G(S, J, P, C, R)$ is computed, where $P$ is the set of paths, $C \subseteq P \times P$ is the compatibility relation, and $R \subseteq P \times P \times L \times L$ represents the connectivity relation between paths according to the intersection places $L=\{H, T, I\}$. Figure 5 shows an example. 


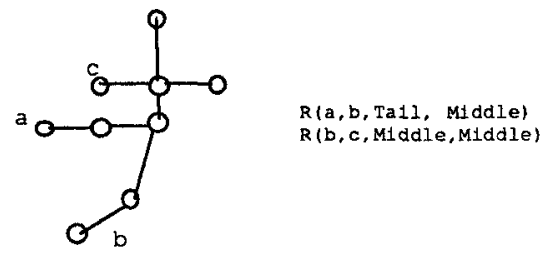

Fig. 5. Relation attributes of three paths.

Intuitively, if two paths are incompatible, and one of them is matched into a true stroke in a model, the other one cannot be matched in the same interpretation because the shared segments should have a single matching.

\section{Triplet Indexing}

We choose indexing or hashing as a object recognition technique to avoid the separated matching with each model. Indexing schemes share a uniform underline structure $[3,6]$ : they compute invariants from the input and then use their index pointers on a look-up table that returns a list of associated models.

Sets of path orientations and their relations are used as invariants. A large range of variability for orientation is allowed and the relations are assumed indispensable for this implementation.

\subsection{Triplets}

A triplet is a set of three connected and compatible paths. A triplet is represented by a vector of 9 entries that encode the relations between the paths. Let $p_{1}, p_{2}$ and $p_{3}$ be three paths and let $R\left(p_{1}, p_{2}, l_{1,2}, l_{2,1}\right), R\left(p_{1}, p_{3}, l_{1,3}, l_{3,1}\right)$, $R\left(p_{2}, p_{3}, l_{2,3}, l_{3,2}\right)$ be the relations between them, where $l_{i, j}$ is the place on $p_{i}$ that intersects $p_{j}, 1 \leq i, j \leq 3$. Then, the triplet is represented by

$$
\left(p_{1}, p_{2}, p_{3}, l_{1,2}, l_{2,1}, l_{1,3}, l_{3,1}, l_{2,3}, l_{3,2}\right) \text {. }
$$

At most two of the paths may not be related: in this case, they are both related to the third path, and the two vector entries for the location of their connection are filled with the symbol NONE.

During index pointer calculation, the angles of the three first entries are quantized into 7 possible values. There are 3 possible values for the first 4 location entries, and 4 possible values for the last two entries including $N O N E$, given a total of $7^{3} \times 3^{4} \times 4^{2}=444,528$ possible index pointers. No other attributes of the paths are used during the look-up, so triplets represent ways of connecting three line segments with a fixed number of possible orientations but disregard their length. Figure 6 shows examples of triplets. Only compatible and related paths 
are considered thus maintaining the number relative low. Experimentally, it was found that on 100 characters, the average number of triplets was 544.2 , which may imply that the number of triplets is less than quadratic in the number of paths.

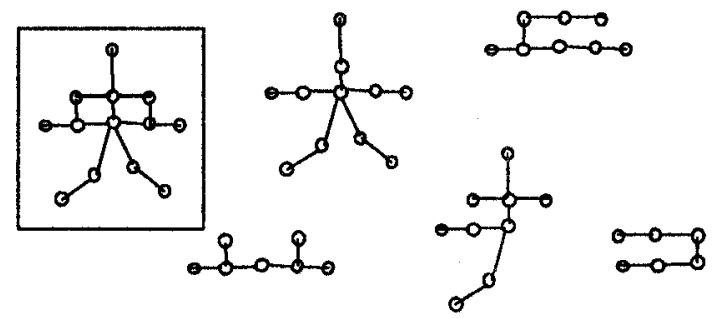

Fig. 6. Some triplets for a skeleton graph.

For each ideal triplet, a big range of variations of the parameters is generated so that the system stores in advance not only ideal model triplets but also their distortions. During triplet storage, all 6 permutations are considered. The second possible variation is the inclination of each path. The quantized angle value is allowed to vary one quantization step in each direction. Therefore, each triplet generates $3^{3}$ entries.

\subsection{Vote Accounting}

During storage, triplets are calculated for models, including their permutations. A hash table entry is a tuple $\left(t, M, h_{t}, w_{t}, c^{(M)}\right)$, where $t$ is a triplet, $M$ is a model, $h_{t}, w_{t}$ are the height and width of the bounding box of the triplet $t$, respectively, in the model coordinate system, and $c^{(M)}$ are the coordinates of the center of the model relative to the top-left corner of the bounding box of $t$. See Figure 7. Thus a table entry includes geometric parameters that are used to recover the vertical and horizontal scale of the triplet in the image and the expected position of the model in the input.

During recognition, triplets are generated in the same way on the input. Given a triplet $t_{0}$, its index is calculated and the list of entries is retrieved from the table. Using the height and width of the bounding box of $t_{0}$, each entry is scaled to the input size, and the center of the triplet model is calculated in the Cartesian base determined by $t_{0}$. Let $c_{0}^{(M)}$ be the model center on the input coordinate system.

A hypothesis is a tuple $\left(t, t_{0}, M, c_{0}^{(M)}\right)$ and we will refer to it by saying that the triplet $t_{0}$ is a vote for the model $M$ on the place $c_{0}^{(M)}$. The number of quantization steps on each axes of the input coordinate system is set to 4 . 


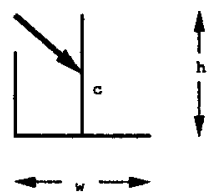

Fig. 7. Attributes of a hash table entry.

Some index-based recognition systems just the number of votes on each (model, position) bucket to decide the most likely of the instance models. However, since triplets are highly structured objects, two votes for the same model should agree on the interpretation of the input parts.

\section{$5 \quad$ Structural Verification}

Given $I\left(S_{I}, J_{I}, P_{I}, C_{I}, R_{I}\right)$ and $M\left(S_{M}, J_{M}, P_{M}, C_{M}, R_{M}\right)$, an input and a model graphs, an interpretation

$$
\Phi: P_{I} \rightarrow P_{M}
$$

is a partial one-to-one mapping such that

- the domain and the range consist of compatible paths, and

- it respects the path relations, $R_{I}$ and $R_{M}$, so that if two paths in $I$ are related on certain locations, its images in $M$ are also related in the same locations.

If $\Phi$ is an interpretation of $I$ in $M$, we also say that $\Phi$ is a shape instance of $M$ in $I$.

The conditions ensure that paths are mapped into paths of the same relative positions. Therefore, an interpretation has the same flavor of homeomorphic subgraphs [8], but it is more relaxed. Distortions are modeled by the possible matchings between alternative subsets of paths and the flexible meaning of the Inside connection of paths.

The purpose of structural verification is to create shape instances from votes in each bucket for a model and a position.

Two votes $v_{1}$ and $v_{2}$, regarded as interpretations, are consistent if their union is an interpretation.

In order to find maximal consistent vote sets, a consistency graph (matrix) is calculated for votes, and maximal cliques [8] in this graph are found by enumerating in increasing size all consistent subsets of votes. Then, the ones that cover the maximum number of segments in the model are returned as the maximal ones. 


\section{Geometrical Verification}

We opt to include knowledge for a great number of possible geometrical arrangements for each model, by training an array of neural nets, one for each structural model. A neural net will learn geometric distributions of matched parts for a particular model. This design has the advantage of being able to include specific knowledge of the particular model, without causing confusion with other models for which other unrelated neural nets are trained.

The feature vector for a neural net associated to a given model includes lengths, inclinations and positions of the paths matched to the model. Firstly, the input horizontal and vertical lengths are scaled to those of the model. For each maximal path in the model, consider the input path matched to it. The following parameters of the input path appear in the input vector: length of horizontal projection $\Delta x$, and length of vertical projection $\Delta y$. The feature vector includes also, for each inside connection on the model path, the relative distance of the actual connection in the input. For example, a 0.4 means that on the input path, an inside connection appears at 0.4 of the path length starting from the path head.

\section{Experiments}

We devise the evaluation procedure described next.

The design, training and development of the system were carried out using the first 100 characters of a data base of printer characters. For evaluation, we took the second 100 characters and recorded manually the number of substructures correctly and incorrectly retrieved, and the number of substructures non retrived that should have been retrieved. Figures 8,9 show recognition of substructures on several characters.

The results are as follows: the system retrieved 674 substructures from 100 Chinese characters. It failed to retrieve 13 substructures ( $98 \%$ retrieval rate). Of the 674 substructures retrieved, 55 were wrong on 18 characters, in the sense that their shape did not correspond to the suggested model ( $92 \%$ precision rate). The average retrieval time was $0.54 \mathrm{cpu}$ seconds per character on a SPARCstation 1.

The most damaging errors are the retrieval failures, because subsequent parts of the system will use the presence or absence of a substructure for final recognition of a full character, but they will not generate new structures. The causes of this type of errors are in order of importance: skeleton generation errors, $31 \%$ (specially, loss of small segments, and direction change); connectivity requirement error, $31 \%$ (paths are not connected as expected); neural net errors, $23 \%$ (the net rejects the generated shape instance), and collinearity grouping errors, $15 \%$ (paths are counterintuitive due to local decisions). The skeleton extraction algorithm may improve by a more precise calculation of stroke lengths. Also, the neural nets should be trained on bigger sets and more carefully, as we explain later. The wrong retrievals have much less importance because they could be pruned easily by subsequent modules. However, it would be better if good 

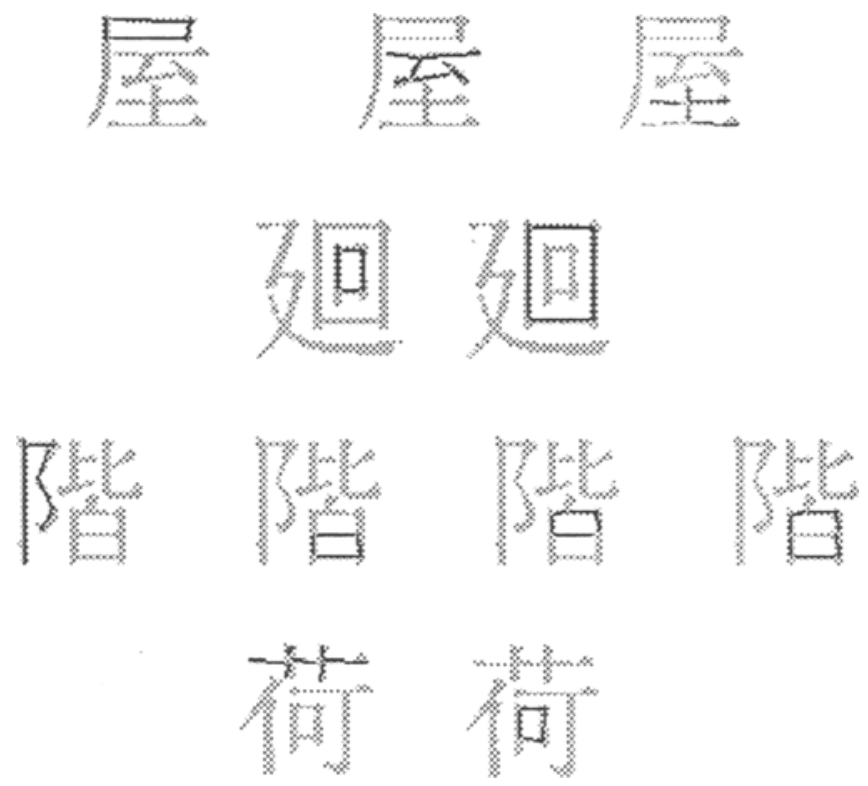

Fig. 8. All substructures recognized for four characters.

substructures do not compete with bad ones. The causes of the errors are as follows: neural net errors, $37 \%$ (better training should have reject geometrically poor shapes); collinearity grouping errors, $31 \%$, lack of use of aspect ratio as a geometric feature, $28 \%$ ((kanmuri) should be wider than high); and skeleton extraction errors $4 \%$.

\section{Conclusions}

It is our purpose with this paper, to show evidence of the possibility to recognize components anywhere on a Kanji string, if all important parts are present and their relations approximately preserved. The system allows a great degree of distortion of the components, both in the geometrical and the structural sense. The geometrical flexibility is obtained by having specific classifiers for each shape, which gives maximal descriptive power for a single shape. The structural flexibility comes from the novel matching of compatible paths that extends the homeomorphic and isomorphic matching of other graph matching algorithms.

Acknowlodgoments. We would like to thank Dr. Joht Vincent, Mr. Thomas Bouton, Dr. Tony Smith and Dr. Hiroshi Sakoh for the useful discussions that give strength to this research. Also to the members of the Document Analysis Group at HCRL for their continuous support. 

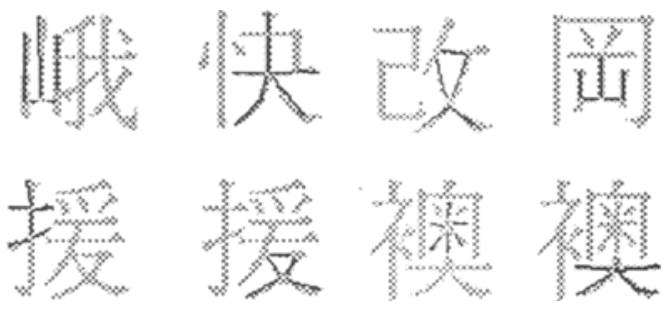

Fig. 9. Several recognized substructures, some of them connected to other parts of the image.

\section{References}

1. N. Babaguchi et al. Identification and extraction of radicals from handprinted Kanji by segment correspondence method. Trans. IECE Japan, PRL, 83(59), 1983.

2. G. Boccignone et al. Recovering dynamic information from static handwriting. Pattern Recognition, 26(3):409-418, 1993.

3. A. Califano and R. Mohan. Multidimensional indexing for recognizing visual shapes. IEEE Trans. PAMI, 6(4):373-392, 1994.

4. R. Cheng, C. Lee, and Z. Chen. Preclassification of handwritten chinese characters based on basic stroke substructures. In The 4th International Workshop on Frontiers in Handwriting Recognition, pages 176-184, Taiwan, RoC, 1994.

5. S. Chou and W. Tsai. Recognizing handwritten chinese characters by strokesegment matching using an iteration scheme. In P. Wang, editor, Character and Handwriting Recognition, volume 30, pages 175-198. World Scientific Series in Computer Science, 1991.

6. S. Dickinson, A. Pentland, and A. Rosenfeld. From volumes to views: An approach to 3-d object recognition. CVGIP: Image Understanding, 55(2):130-154, 1992.

7. H. Fujisawa and K. Marukawa. Full-text search and document recognition of japanese text. In 4 th International Symposium on Document Analysis and Information Retrieval, Las Vegas, Nevada, April 1995.

8. F. Harary. Graph Theory. Addison-Wesley, 1972.

9. X. Huang, J. Gu, and Y. Wu. A constrained approach to multifont chinese character recognition. IEEE trans. on PAMI, 15(8):838-843, 1993.

10. G. Lorette and $Y$. Lecourtier. Is recognition and interpretation of handwritten texts a scene analysis problem? In Proc. of $3 d$ Intern. Workshop on Frontiers in Handwiting Recognition, pages 184-196, Buffalo, New York, May 1993.

11. S. Lu, Y. Ren, and C. Suen. Hierarchical attributed graph representation and recognition of handwritten chinese characters. Pattern Recognition, 24(7):617-632, 1991.

12. S. Mori, C. Suen, and K. Yamamoto. Historical review of OCR reseach and development. In IEEE Proceedings, volume 80, pages 1029-1058, July 1992.

13. J. Rocha and T. Pavlidis. A shape analysis model with applications to a character recognition system. IEEE trans. on PAMI, 16(4):393-404, Apr 1994. 